\title{
Spurious papilloedema and visual field contraction after head trauma ${ }^{1}$
}

\author{
ROBERT T. DALE AND MILES A. GALIN \\ From the Departments of Neurology and Ophthalmology of the New York Medical College
}

An ophthalmoscopic picture indistinguishable from that seen with papilloedema secondary to increased intracranial pressure may occur as a consequence of other conditions. Kestenbaum (1961) has referred to such situations as 'spurious' papilloedema because they do not reflect an elevated intracranial pressure. The following two cases describe instances of spurious papilloedema which were associated with peripheral field contraction, resulting most probably from indirect injury to the optic nerve after head trauma.

\section{CASE 1}

A 20-year-old Puerto Rican youth was admitted to the Metropolitan Hospital Division of the New York Medical College for observation after sustaining a fall on the back of his head during a neighbourhood street baseball game. An occipital laceration was repaired in the emergency room. During the 24 hours after the trauma, he experienced some nausea, vertigo, intense headache, and mild blurring of vision. These symptoms abated about one day later. There was no loss of consciousness or confusion following the incident.

Neurological examination at the time of admission revealed a mentally clear youth who was orientated in all spheres and who remained so throughout his hospitalization. There was no drowsiness or mental obtundation. The pulse was 100 per minute, BP $130 / 70 \mathrm{~mm} \mathrm{Hg}$, temperature $99^{\circ} \mathrm{F}\left(37 \cdot 2^{\circ} \mathrm{C}\right)$, and respiration 18 per minute and of normal character. There was no posthyperventilation apnoea. The neurological examination was essentially normal in all respects except for ophthalmoscopic examination and the visual fields, the latter two examinations being carried out within the first 12 hours of admission.

Visual acuity was 20/20 in both eyes without correction. Visual field examination with the Goldmann perimeter revealed a consistent pattern of peripheral field contraction (Figs. 3 and 4). There was no evidence for malingering. Ophthalmoscopic examination revealed hyperaemia of both discs with bilateral blurring of the disc margins. Venous congestion was marked but spontaneous venous pulsations were present in both ${ }^{1}$ Aided by USPHS grant NB07162-03, a grant from the Susan Greenwall Foundation, and Neurology Training Grant 2T01 NB-05376-07 from the National Institute of Neurological Diseases and Blindness. eyes. In the right eye, there was a haemorrhage at the 듬 lower nasal disc margin (Fig. 1). In the left eye, a smaller $\frac{\bar{N}}{\bar{D}}$ haemorrhage was noted on the surface of the disc at $\mathbb{Q}$ three-four o'clock (Fig. 2). The maculae appeared normal. Routine radiographs of the skull revealed fractures $क$ in the right occipital and left temporal regions with no $\vec{\circ}$ evidence of depression. Tomograms of the optic canals and orbits revealed no fractures. Despite the ophthalmo- $\vec{\omega}$ scopic appearance of papilloedema, the patient showed 2 no clinical evidence of increased intracranial pressure, $\vec{\exists}$ and a lumbar puncture was performed shortly aftero admission. The opening pressure was $170 \mathrm{~mm} \mathrm{H}_{2} \mathrm{O}_{\omega}^{\omega}$ and the closing $160 \mathrm{~mm} \mathrm{H}_{2} \mathrm{O}$. The fluid was milds iv xanthochromic. The protein was $39.9 \mathrm{mg} \%$, glucose $71 \mathrm{mg} \%$; there were no cells. A brain scan was norm\&. Electroencephalography was normal.

Serial visual field examinations revealed no change? throughout his hospitalization. His fundi improved with resolution of the haemorrhages and disc blurrifer after one to two weeks after which some paleness if the discs was noted. He has been scheduled for ouf $\vec{\theta}$

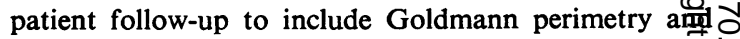
fundus photos.

\section{CASE 2}

A 36-year-old male was seen for ophthalmoscopic consultation in March 1965, because of migraine head- $\mathbb{D}$ aches. Visual fields at this time were entirely normal (Fig. 5). In September 1965, the patient was involved in a street brawl in New York City and suffered a concussion. He was unconscious for only a brief period and, except for mild confusion immediately after the incident, was mentally clear thereafter. Nausea, vertigo, headache, blurring of vision, or other symptoms were denied.

After he had recovered from the concussion, the results of neurological examination were within normal limits with the exception of ophthalmoscopic examination and visual fields. There was hyperaemia of both discs with bilateral blurring of the disc margins. Bilateral음 venous congestion was noted, but venous pulsations $>$ were present in both eyes. There were no haemorrhages. 을 The maculae appeared normal. Visual acuity was $20 / 20$ in both eyes. Visual field examination done one day $\widetilde{N}$ after the injury showed general contraction of the fields $N$ (Fig. 6). All attempts to infer malingering failed. Fields $N$ at $2 \mathrm{~m}$ were of the expected ratio to the $1 \mathrm{~m}$ field. 


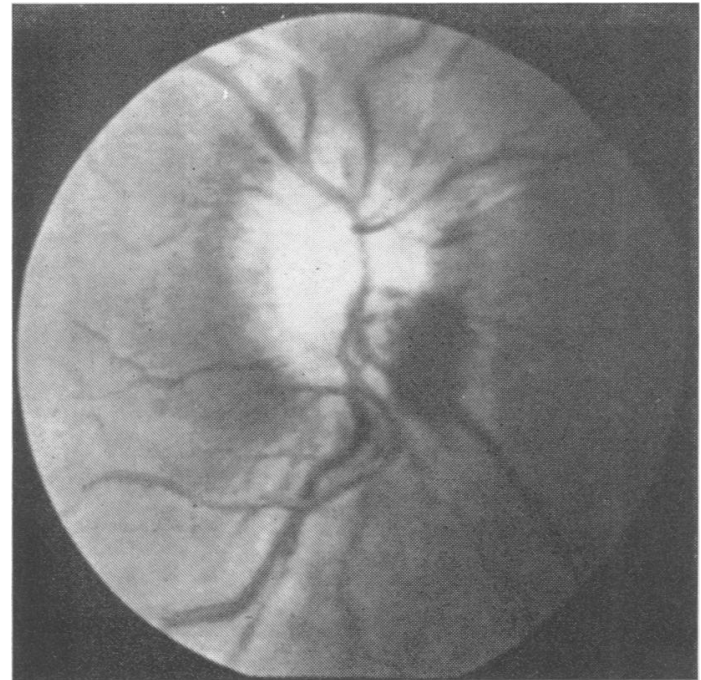

FIG. 1. Right fundus of case 1. Note haemorrhage at the nasal margin and blurring, particularly at the superior and inferior aspects of the disc.

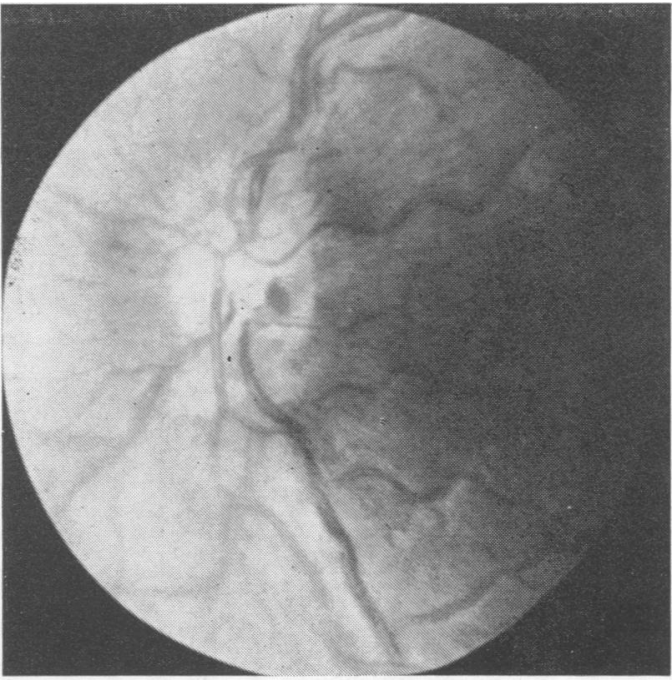

FIG. 2. Left fundus of case 1. Note haemorrhage on the disc surface at 4 o'clock, blurring of disc margins, and venous tortuosity.
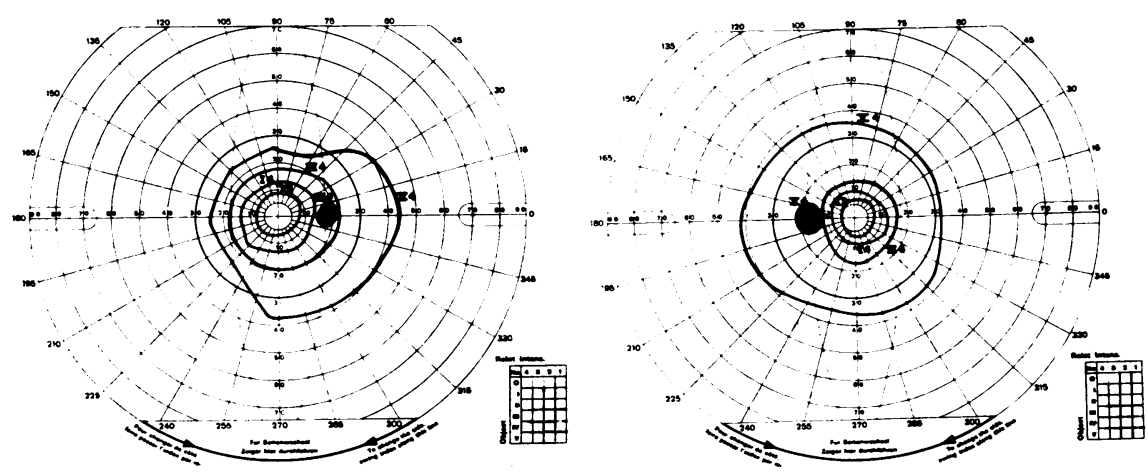

FIG. 3 (Left). Visual field $O D$ of case 1 with Goldmann perimeter. Note peripheral contraction, which is most marked in the nasal field.

FIG. 4 (Right). Visual field $O S$ of case 1 with Goldmann perimeter. Again, note general peripheral contraction.


FIG. 5. Bilaterally normal visual fields of case 2 obtained before head injury. ' $A$ ' represents $2 / 1,000 \mathrm{~W}$.
Radiographs of the skull including special views of the optic canals and orbits revealed no evidence for fracture. An EEG was normal. A lumbar puncture done shortly after admission revealed an opening pressure of $180 \mathrm{~mm} \mathrm{H}_{2} \mathrm{O}$. The protein was $36 \mathrm{mg} \%$ and there were no cells. The fluid was clear.

Throughout the patient's hospitalization and after his discharge, he remained asymptomatic. The papilloedema began to recede after several days and the discs became pale. The progress of the patient's visual fields was followed in the out-patient clinic where they continued to show the same amount of constriction, and for three years after the incident have remained essentially unaltered (Fig. 7). 


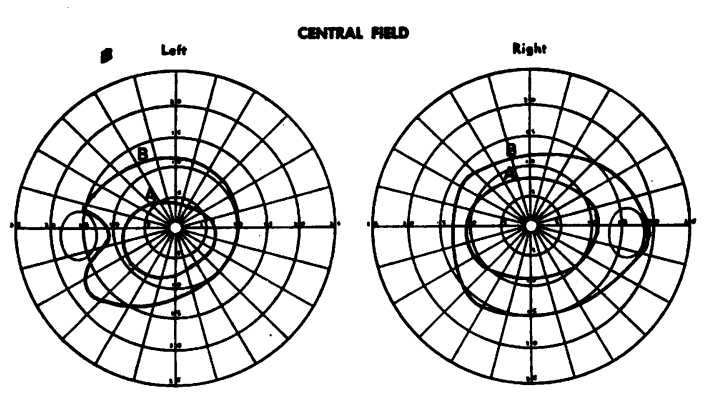

FIG. 6. Visual fields of case 2 obtained within 24 hours after head trauma. ' $A$ ' indicates $2 / 1,000 \mathrm{~W}$; ' $B$ ' is 20/1,000 $W$. Note general peripheral contraction of both fields of vision.

\section{DISCUSSION}

Spurious papilloedema is 'true' papilloedema in that the two conditions are indistinguishable ophthalmoscopically. A normal CSF pressure in spurious papilloedema is the distinguishing feature. In both of these cases which followed head trauma, the CSF pressure was normal throughout the hospital course by direct measurement and, even more crucially, by the clinical symptoms and signs. There was no evidence of diencephalic compression which could be caused by tentorial herniation such as mental obtundation, respiratory changes, or pupillary abnormalities. Hence, these cases seem to be genuine examples of spurious papilloedema as defined by Kestenbaum (1961), and do not appear due to the rapid development of cerebral oedema.

It is believed that the papilloedema seen in these patients was the result of indirect injury to the optic nerve. The failure to demonstrate any fracture into the optic canal in these patients including tomograms militates against the possibility of direct injury to the optic nerve. It has been demonstrated that disturbance of vision in one or both eyes occurs in 0.5 to $1.5 \%$ (Turner, 1943; Rowbotham, 1964) of head injuries where there is no evidence of direct damage to the optic nerves. In these cases, absence of skull fracture of any type, and, in some instances, post-mortem examination, has confirmed that the injury was indirect.

Reports on the frequency of papilloedema after indirect injuries to the optic nerve have not shown consistent agreement. It has been stated by certain authors (Evans, 1929; Rodger, 1943) that no changes are to be observed in the optic discs of such patients, even including those who later developed post-traumatic optic atrophy after their indirect optic nerve injury. Turner (1943), in a series of 46 cases of indirect injury to the optic nerve, states that two cases examined in the first

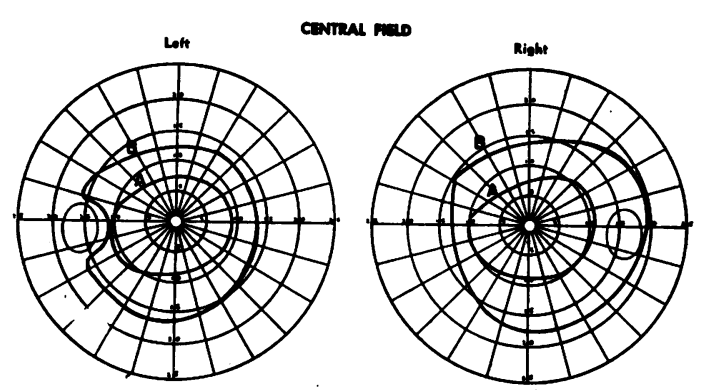

FIG. 7. Visual fields of case 2 performed approximately three years after the head injury. ' $A$ ' indicates $2 / 1,000 \mathrm{~W}$; ' $B$ ' is 20/1,000 W. Little change has occurred from. fields obtained several hours after the head injury as shown in Fig. 6.

few days after the injury showed some scattered haemorrhages in the retina. He thought that these were the result of direct trauma to the globe and were not directly related to the optic nerve injury. It may be significant that, in Turner's series, it is not specified that all patients received ophthalmove scopic examination early in their hospitalization Hooper (1951), who examined 10 patients in th8 8 acute stages of injury in a series of 58 patients with indirect optic nerve injuries, noted changes in the fundus in eight. The changes described were similar to those seen in the patients reported in this paper and included congestion of the veins. blurring of the disc margins, and hyperaemia of the disc. The disc changes in Hooper's patients persisted for at least three to seven days and then receded. In some of the cases, post-traumatic optic atrophy began to appear within three to five weeks. Blakeslee (1929) also noted disc changes in the early stages of many cases of indirect injury to the optic nerve. Swelling of the retinal vessels accompanied by oedema with obliteration of the disc margins was noted in 18 of his cases. These cases were from a series of 610 cases of skull fracture without direct involvement of the visual pathways by the fracture.

In indirect injuries to the optic nerve, a variety of types of visual loss ranging from none to complete blindness have been described. When blindness occurs, it may be immediate or delayed. Although unilateral visual loss is more common, bilateral loss may occur as well. In some cases of immediate visual loss, recovery began after two to three days. According to Walsh (1966), cases of immediate and permanent visual loss are more likely to be due to indirectly produced tears or to contusion necrosis of the optic nerve based upon shearing forces resulting from the injury. It is of 


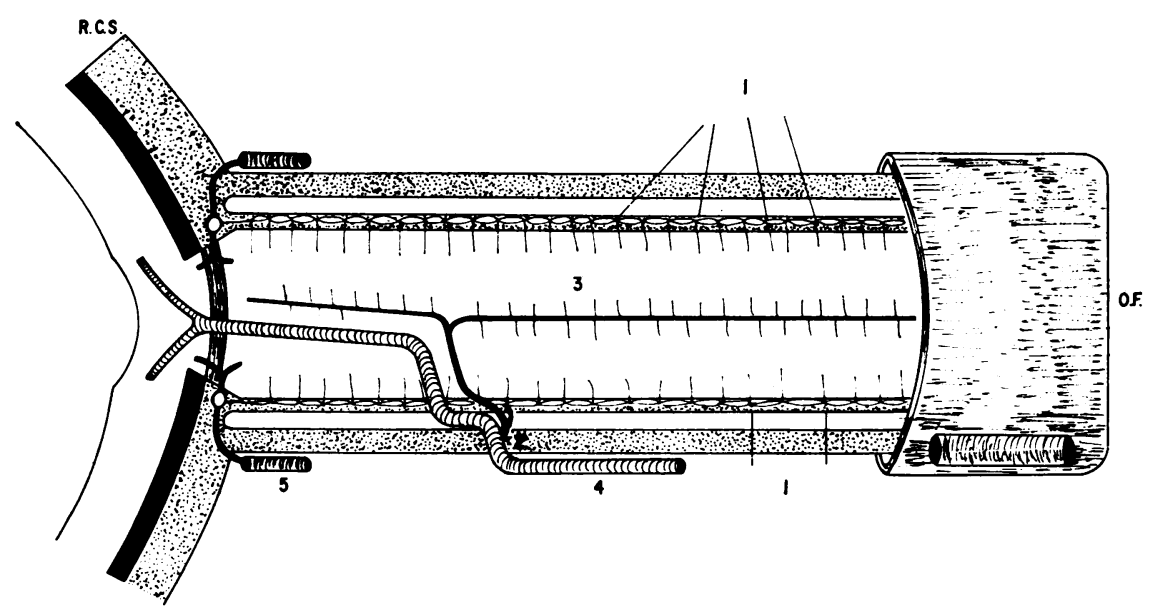

FIG. 8. Blood supply io optic nerve. O.F.: optic foramen; R.C.S.: retina, choroid, and sclera; 1 : pial vessels; 2 : recurrent collateral supply to inferior pial vessels from central retinal artery; 3: posterior central artery of Kuhnt to macular fibres; 4: central retinal artery; 5: posterior ciliary artery. Note that the recurrent collateral pial supply from the central retinal artery is predominantly to the inferior aspects of the nerve, which may be one factor in the frequent sparing of the superior peripheral fields in indirect optic nerve injuries (see text).

interest that both Walsh (1966) and Rowbotham (1964) agree that patients with delayed visual loss should be subjected to unroofing of the optic canal in an attempt to save any useful vision. In cases of immediate blindness such operations have been found unrewarding. Therefore, because of the obvious therapeutic importance of the state of vision immediately after injury, Walsh has suggested the use of evoked cortical potentials as well as evaluation of the pupillary light response to assist in the evaluation of unconscious patients with head trauma.

Field defects in indirect optic nerve injuries may consist of scotomata, peripheral defects, or both. The peripheral field defects, which are the more common, consist of all types ranging from a complete temporal or inferior hemianopia, to small sector defects, to general contraction with one part of the field more involved than the rest. Fading edges are typical. The two patients discussed in this report fall into the latter category, in that they show general constriction of their visual fields with one patient showing greater involvement of the inferior fields and, in one eye, the nasal field. In the second case described, the peripheral contraction can be regarded as a permanent defect in that it has been present without change for three years. Although he was not followed for as long a period, permanent contraction appears likely in the first patient as well.
Walsh (1966) has classified indirect optic nerve injury on the basis of primary and secondary lesions. Among primary lesions, he includes haemorrhages into the optic nerve sheaths, the nerve, and the dura. Secondary lesions are those resulting from disease at a distal site-that is, thrombosis or circulatory failure. The area of the nerve most frequently involved in primary lesions is in the posterior orbit, the optic canal, or the intracranial nerve. Walsh, Turner, and Rowbothan concur that, among these locations, the most common site of indirect injury is within the optic canal itself. The mechanism of such an injury and the reason for its locus in the optic canal portion of the nerve can be related to the anatomy of the optic nerve and its blood supply (Last, 1968). The optic nerve consists of three parts, an intraorbital portion of about $25 \mathrm{~mm}$, a canalicular portion of about 4 to $10 \mathrm{~mm}$, and an intracranial portion of about $10 \mathrm{~mm}$. The intracanalicular portion is supplied by the ophthalmic artery. However, the macular and the peripheral fibres of the intracanalicular portion of the optic nerve, although supplied by the ophthalmic artery, are supplied by different branches of that artery. In most instances, the macular fibres are supplied by a recurrent artery, the posterior central artery of Kuhnt. This artery is a branch of a collateral artery coming from the central retinal artery. It passes backward in the nerve to supply the macular bundle up to and 
including the intracanalicular portion. The peripheral optic nerve fibres in the intracanalicular portion are supplied by small, direct branches from the ophthalmic artery which pierce the dura on the various aspects of the nerve but predominantly above and at the sides. The fewest number of vessels pass in from below. However, this portion has additional collateral supply which is also derived from recurrent branches of the central retinal artery (Fig. 8). Thus, the intracanalicular portion, in contrast with the orbital and intracranial parts of the nerve, has a relatively meagre vascular supply to its pial network, particularly in its superior and lateral aspects where there is little recurrent collateral flow.

Perhaps an even more significant reason for the predominance of injuries in the intracanalicular locus of the nerve is that the dura is here anchored to the bone and to the pia. This forms a point for fixation of the nerve. Schwalbe held that this point of fixation was chiefly in the superior portion of the nerve within the canal. A fall on the face and less commonly on the temporal or occipital portions of the head could result in a stretch of the optic nerve either from the motion of the eyeball itself or from the shearing stress of the brain moving faster than the nerve. The nerve would then shift relative to its membranes and, at the point of greatest fixationthat is, within the optic canal-rupture of the small vessels within the pial network could occur. This would be followed by local haemorrhage, thombosis, necrosis, and swelling of the nerve. Central vision would not be affected unless the injury were sufficiently extensive to involve the distribution of the recurrent posterior central artery of Kuhnt. Also, the superior fields would be relatively spared, as is common with indirect optic nerve injuries, since there is more extensive collateral flow in the inferior portions of the nerve. If the distribution of the recurrent artery of Kuhnt is involved, scotomatous defects may occur which, although not present in the cases reported in this paper, have been noted in other patients.

Behrman (1966) and others (Gifford, 1886; Kyrieleis, 1929) have adduced evidence that obstruction to perivascular papillo-orbital lymph channels within a swollen nerve is one way of accounting for the development of papilloedema. The local swelling, caused by the mechanism just described, could lead to obstruction of such lymph channels within the nerve itself. In addition to obstruction to lymph channels within the nerve, haemorrhage into the dural optic nerve sheath may result in a haematoma around the nerve producing obstruction of intradural lymph channels. Pringle (1916) and others have shown that such subdural haematomas of the optic nerve sheaths are not unusual in indirect optic nerve injuries. As has been noted, papilloedema and visual loss are not always mutual concomitants of indirect optic nerve injuries. Presumably visual loss would be more likely from local haemorrhage, thrombosis, and oedema within the nerve than from haematoma formation surrounding it. A dural optic nerve sheath haematoma could obstruct the intradural sector of the papillo-orbital lymph channel and result in papilloedema without necessarily producing visual loss. In cases with visual loss and papilloedema, both sites of obstruction (from swelling within the nerve itself and from haematoma within the optic nerve sheath) may be important. However, when papilloedema is present without visual loss, it would seem more likely to be due to obstruction of the intradural portion of the papillo-orbital channel by haematoma formation.

Apart from the pathophysiological explanation for the mechanism of the injury, the recognition of this entity underscores the importance of overall, complete neurological examination of patients with head injury for evidence of increased pressure rather than relying on a single sign such as papilloedema which, in some cases, may not indicate elevated intracranial pressure. Further, its recog- $\stackrel{\mathbb{\otimes}}{\Omega}$ nition is important from the standpoint of clinical management, since it obviates the performance of inappropriate radiological contrast procedures in such patients and suggests the importance of other special radiographic studies, such as tomograms of the optic canals, to make certain fracture is not contributing to the pathology.

\section{SUMMARY}

Two patients with bilateral papilloedema after head trauma without increased intracranial pressure are discussed. Bilateral contraction of the visual fields, apparently on a permanent basis, was noted in both patients. The pathophysiology is believed to be related to indirect injury to the optic nerve, which has been reported as occurring in 0.5 to $1.5 \%$ of head injuries, though not in all cases with papilloedema. However, evidence from the literature is presented suggesting that, when ophthalmoscopic examination is done early, spurious papilloedema with indirect optic nerve injuries may be more frequent than is commonly appreciated. The mechanism appears to be related to the nature of the vascular supply to the optic nerve. The recognition of this phenomenon has obvious clinical importance in obviating unnecessary radiological contrast procedures in patients with head injuries. 


\section{REFERENCES}

Behrman, S. (1966). Pathology of papilloedema. Brain, 89, 1-14.

Blakeslee, G. A. (1929). Eye manifestations in fracture of the skull, Arch. Ophthal., 2, 566-572.

Evans, J. J. (1929). The ocular manifestations of head injuries. Trans. Ophthal. Soc. U.K., 49, 342-344.

Gifford, H. (1886). The direction of the lymph-streams in the eye. Arch. Ophthal., 15, 153-162.

Hooper, R. S. (1951). Orbital complications of head injury. Brit. J. Surg., 39, 126-138.

Kestenbaum, A. (1961). Clinical Methods of Neuro-Ophthalmologic Examination. 2nd edn. Grune and Stratton: New York.

Kyrieleis, W. (1929). Uber Stauungspapille. Klinische, anatomische und experimentelle Untersuchungen. Albrecht v. Graefes Arch. Ophthal., 121, 560-638.
Last, R. J. (1968). Wolff's Anatomy of the Eye and Orbit. 6th edn. Lewis: London.

Pringle, J. H. (1916). Monocular blindness following diffused violence to the skull: its causation and treatment. Brit. J. Surg., 4, 373-385.

Rodger, F. C. (1943). Unilateral involvement of the optic nerve in head injuries. Brit. J. Ophthal., 27, 23-33.

Rowbotham, G. F. (1964). Acute Injuries of the Head. Williams and Wilkins: Baltimore, Md.

Turner, J. W. A. (1943). Indirect injuries of the optic nerve. Brain, 66, 140-151.

Walsh, F. B. (1966). Pathological-clinical correlations I. Indirect trauma to the optic nerves and chiasm. Invest. Ophthal., 5, 433-442. 